\title{
Dos años de Jefatura de Clínica en el Instituto de Protección Materna e Infantil "Concepción Villaveces de Acosta"
}

\author{
Por el doctor Fernando Mera González \\ Trabajo para el concurso de agregación en la Cátedra de Clínica \\ Obstétrica de la Facultad de Medicina de la Universidad Nacional de \\ Bogotá. - 1954.
}

\section{PARTO ESPONTANEO}

Número total de partos: 3.545 .

Número de partos a término: 2.835 .

Número de partos prematuros: 710 .

Número de partos intervenidos: 426.

Número de partos espontáneos: 3.119 .

Fueron frecuentes las alteraciones de la contracción uterina motivadas por las inercias primitivas o secundarias, y se estableció el tratamiento médico con ocitócicos a pequeñas dosis. Se utilizó el Pitocín, diluído en agua, para inyectar dosis fraccionadas en espera de la respuesta, teniendo en cuenta la susceptibilidad especial de cada paciente a la droga. En varias ocasiones los trastornos de hipertonía y exageración en la intensidad o frecuencia de las contracciones uterinas, motivaron medicación sedante de la fibra, para normalizar la dinámica alterada. Debo cestacar la utilización en nuestro medio de dosis altas de principio ocitócico en una sola inyección, a mujeres que iniciaban su parto normalmente en sus casas. Otras veces se provocó el edema y la infección del cuello, impidiéndose la dilatación cervical, por los tactos y manipulaciones vaginales de enfermeras o comadronas ajenas al servicio que, por su ignorancia, pretendieron acelerar la expulsión del feto inyectando hormona retrohipofisaria. dando ocasión a la tetanización de la fibra con inminencia de ruptura del órgano y consiguiente muerte del feto. 
Tal era el estado de la parturienta, cuando acudía al hospital, en demanda de los servicios médicos. Claro está que, en esas condiciones, se buscaba sedar y normalizar la contracción uterina para realizar el parto por las vías naturales en cuanto fuera posible o intervenir obstétricamente para salvar la vida de la madre, perdida, como ya estaba, la del hijo.

Se tuvo especial cuidado en el tiempo de duración total del parto, y se resolvió la conducta apropiada de acuerdo con la distocia. Los espasmolíticos se utilizaron en los trastornos funcionales del cuello, cuando la dilatación del orificio se veía dificultada a pesar de la buena contracción del cuerpo. La dilatación digital cuidadosa del cuello se hizo procurando no desgarrar el tejido y para ello se intervenía únicamente después del borramiento o sea con bordes blandos y fáciles y cuando el orificio adquiría una dilatación apreciable, siempre superior a los 5 centímetros. Ello con el fin de lograr éxito con la intervención, porcue en la práctica he observado que intentar dilatar un cuello grueso y de escasa dilatación es provocar desgarros disimulados del cervix, que más tarde traen consecuencias funestas para los partos posteriores, por las lesiones de cicatrización que entrañan y por las cervicitis y ulceraciones con leucorrea abundante fuéra del estado puerperal.

Para la dilatación digital, se tuvo en cuenta únicamente la detención del parto, con presentación cefálica encajada por distocia del cuello, el cual cedía clínicamente a las maniobras manuales que, asociadas a la acción de los espasmolíticos, aceleraban por las vías naturales el parto según indicación materna o fetal.

Los desgarros perineales que experimentaron las pacientes hospitalizadas en el servicio, fueron cuatrocientas sesenta y dos en total, lo que da una incidencia de $15.22 \%$, cifra alta si se tiene en cuenta que más valdría una episiotomía de bordes quirúrgicos, y por lo tanto, regulares y de fácil afrontamiento, que un desgarro de bordes anfractuosos e irregulares de difícil reparación. Por lo demás, el desgarro obedece al concepto errado del obstetra, que no supo prever la posible aparición de lesiones traumáticas del periné y permitió el estallido de los tejidos con prolongación en la duración del parto, cuando una episiotomía habría facilitado la expulsión del feto, con evolución posterior más normal en relación con la integridad reparadora de los diversos planos perineales. El número de episiotomías fue de trescientos sesenta y dos, con un porcentaje de $12.34 \%$, cifra insuficiente si 
se tiene en cuenta que en la primípara se aconseja el uso sistemático del procedimiento, y que la intervención es favorable y ventajosa para conservar las relaciones anatómicas de los tejidos y para favorecer la expulsión fetal.

No hallo explicación satisfactoria capaz de justificar la demora en el parto por el deseo de conservar íntegramente el periné, cuando lógicamente los tejidos de la vagina y vulva tienen que distenderse al máximo para permitir el paso de un feto a término, dejando lesiones y rupturas microscópicas por el vencimiento de las fibras en su limite de elasticidad, sobre todo en las primíparas. Por otra parte, considero que se justifica la función cel médico de lograr la rápida evacuación del contenido uterino y no esperar indefinidamente la distensión de los tejidos a expensas de mayor deformidad cefálica y con mayor peligro en la vitalidad fetal.

\section{PRESENTACION DE PELVIS}

Presentación de pelvis completa (incluyendo diecinueve embarazos gemelares) : 100 .

Presentación de pelvis incompleta (incluyendo siete embarazos gemelares) : 50 .

Frecuencia de pelvis completa: $3.21 \%$.

Frecuencia de pelvis incompleta: $1.60 \%$

Debemos considerar el parto de pelvis en la primípara y en la multípara que fue sometida ya a la prueba de propercionalidad de su cinturón óseo con los diversos polos del feto. También varía el pronóstico fetal, si se atiende a la modalidad de presentación pelviana, bien entendido que el parto es más favorable en la pelvis completa. Otro de los factores de importancia en las primigestantes es la edad, ya que, cuando llegan a los treinta o más años, surgen nuevas causas de distocia: ruptura precoz de las membranas que dificulta la diltación cervical, inextensibilidad cie las articulaciones, que retienen la cabeza última provocando la muerte del feto. Por tales razones, recomendé la cesárea en todos aquellos casos de presentación de pelvis en primigestante añosa, previo diagnóstico de un parto difícil o imposible por las vias naturales. Si se trataba de una presentación de pelvis, completa o incompleta, en primigestante no añosa, después de examinar detenidamente las debidas proporciones entre los diámetros pelvianos y el tamaño aproximado del feto, vigilaba el trabajo para lograr un parto espontáneo, siempre y cuando la dilatación 
del cuello fuera normal y la fuerza impulsora hiciera descender la presentación a gran coronamiento, circunstancias en las cuales hacía una episiotomía paramediana que facilitaba posteriormente el descenso de los brazos y la extracción de la cabeza por maniobra de Mauriceau. Cuando en el transcurso del parto surgia un espasmo del cuello, que no cedía al tratamiento espasmolítico, o cuando se hacía inminente el sufrimiento fetal por trabajo prolongado, nunca insistí en completar la diltación del orificio por maniobras digitales, por estimar que ello es inconveniente, sino que, tratándose de primigestantes, aconsejaba la cesárea segmentaria.

Otro es mi modo de pensar con referencia a las multíparas, en que la presentación de pelvis completa o incompleta puede dejarse evolucionar libremente, y el médico ser más amplio en la conducción del parto sin intervención quirúrgica. A mis colaboradores en el servicio les indiqué diversas normas de tranquilidad para la atención de los partos con presentación de pelvis: no precipitarse en las maniobras de extracción fetal y reservar la intervención para los casos en que ella sea estrictamente necesaria. La naturaleza es sabia. La función médica es subsiciaria y de ayuda.

Cuando el feto muere en la presentación de pelvis, la conducta se simplifica, porque en estas condiciones se puede permitir la evolución espontánea del parto o buscar la extracción del feto por descenso del pie, del cual se tracciona con suavidad y en forma intermitente hasta lograr la dilatación completa del cuello. Se indicará la basiotripsia en las retenciones de la cabeza última. por desproporeión cefalopélvica.

En relación con el parto de pelvis y sus peligros, es muy ilusrativo el caso de una multípara a término y feto en presentación ce pelvis completa, en que el parto evolucionó fácilmente hasta la extracción de la cabeza última por maniobra de Mauriceau. En este momento, y acaso por la insuficiente dilatación del cuello, se produjo sobre el ángulo izquierdo un desgarro, que se prolongó al segmento en una extensión de 5 centímetros. El diagnóstico se hizo introduciendo la mano dentro del útero para provocar el alumbramiento por haberse hecho imposible en otra forma. Practiqué una histerorrafia segmentaria en el sitio del desgarro, lográndose el éxito materno y fetal.

La referida experiencia, entre otras, pone de presente el peligro a que está sometida una paciente en el parto pelviano por la desgarradura del cuello, que puede a veces extenderse al seg- 
mento inferior. Desde el punto de vista materno, porque el pronóstico fetal es reservado dada la frecuente dilatación incompleta del cuello, que retiene los brazos propiciando su ascenso y motivando lesiones sobre ellos a más de retenciones de la cabeza última que exponen al feto a la asfixia.

\section{PRESENTACION DE CARA}

Número de partos en presentación de cara: 19.

Partos en presentación de cara intervenidos con forceps: 2. Partos en presentación de cara intervenidos por cesáreas: 8. Partos espontáneos en presentación de cara: 9. Frecuencia de la presentación de cara: $0.53 \%$. Mortalidad fetal en la presentación de cara: 1 .

La conducta en la presentación de cara varía con múltiples factores, a saber: a) Variedad de posición, ya que las mento-posteriores persistentes hacen imposible el parto por las vías naturales; b) Multiparidad, que hace favorable la mutación o la evolución y rotación de la presentación a la extremidad anterior de los diámetros maternos, y c) Poder de contracción de la fibra muscular uterina, que impulsa el feto a través del cinturón óseo normal para lograr el parto espontáneo.

Cuando una presentación de cara se adapta sobre el estrecho superior por uno de los diámetros transversos y en el transcurso cel parto verifica la rotación anterior, se podrá esperar la libre evolución del trabajo, pues si los diámetros pelvianos guardan la debida proporción con el polo cefálico, seguramente se realizará el parto espontáneo. Empero, si la rotación se dificulta o hay tendencia a la posición mento-posterior, no se efectúa el encajamiento o el parto se detiene por enclavamiento de la presentación.

La primiparidad en la presentación de cara hace difícil las diferentes maniobras obstétricas; la mutación se imposibilita por li tonicidad de las paredes uterianas y la aplicación del forceps es más laboriosa y peligrosa para la madre y el feto. En cambio, la multiparidad facilita la dilatación cervical y favorece el descenso de la presentación, lo mismo que las intervenciones a que da lugar la presentación de cara.

En esta modalidad de presentación, sobreviene un aumento cel poder contráctil de la fibra muscular, que repercute sobre la ¿uración total del trabajo, prolongándolo y ocasionando diferen- 
incapaz de vencer el obstáculo que ofrece la cabeza fetal deflejada. Otras veces, y es lo más frecuente, el útero se contrae en exceso y se distiende el segmento produciendo la ruptura del órgano. La prolongación en el parto motiva el sufrimiento fetal con aumento de la morbilidad y mortalidad del hijo.

La mutación por maniobras internas resulta eficaz en las multíparas, cuando se intenta con cuello enteramente dilatado y bolsa de las aguas aun íntegra, que permiten la introducción de la mano para asir el occipital y buscar su descenso hasta adaptar ei diámetro suboccípito bregmático al estrecho superior, haciendo cambiar la presentación de cara en una de vértice, ésta si favorable a la evolución espontánea del parto. La mutación por maniobras externas se puede lograr, en una gran multípara, a favor de las paredes abdominales y uterinas flácidas y habiendo líquido amniótico suficiente dentro de la cavidad. De lo contrario, el fracaso es inevitable.

El forceps tiene su indicación cuando el cuello, completamente dilatado o fácilmente dilatable, permite la introducción de las cucharas para una variedad de posición anterior o transversa (para la posterior no es aconsejable) con presentación de cara encajada profundamerte y sin rotación ni descenso difíciles. Tan absurdo sería hacer una aplicación de forceps para una cabeza sin encajar o móvil, aunque no haya desproporción cefalopélvica, como intentarla para una variedad posterior en busca de la rotación y el descenso; con ello únicamente se consigue el traumatismo violento de la cabeza fetal, de consecuencias funestas para la vida del niño en el supuesto remoto de que éste no muera duseante el acto operatorio.

La versión interna la contraindiqué siempre en toda presentación de cara, por considerar dicha maniobra obstétrica, peligrosa para la madre y para el feto, y por creer que es más favorable y da mayor seguridad en estos casos la cesárea segmentaria.

Las sinfisiotomías y pubiotomías son inconvenientes para la madre, ya que son operaciones ciegas, que entrañan grave peligro de traumatismo para los órganos vecinos, y ofrecen, desde el punto cie vista funcional de la mujer, resultados poco satisfactorios, al contrario de lo que ocurre con la cesárea abdominal.

La cesárea segmentaria transperitoneal es la intervención indicada en las variedades posteriores persistentes o cuando se dificulta el encajamiento de la presentación en la anterior o la transversa. Tiene especial indicación en la primigestante que es la que mayor cuidado y atención requiere en la vigilancia del parto. 
La conducta que debe seguirse en la presentación de cara, varía según las relaciones que guarde el mentón con los diámetros anteriores o posteriores maternos; pero el parto se dejará evolucionar libremente cuando encaje la presentación por uno de los diámetros anteriores y a condición de que el trabajo no se prolongue extraordinariamente y no sobrevengan nuevas causas de distocia.

El forceps está indicado principalmente en las multíparas, cuando se encaja la presentación con demora del descenso en variedad mento-anterior o mento-pública y cuando hay dilatación completa del cuello.

En todos aquellos casos en que falta encajamiento, existe rotación pervertida del mentón hacia atrás, se presentan signos de sufrimiento fetal, trabajo prolongado o dificultad aparente para el parto, la cesárea es la operación aconsejada, lo mismo que cuando se agrega una distocia uterina, fetal u ovular, a la presentación de cara.

\section{PRESENTACION DE FRENTE}

Número total de presentaciones de frente: 11.

Frecuencias: $0.31 \%$

Esta presentación es francamente distócica y cuando el feto es de término el parto se hace imposible por las vías naturales, aunque la pelvis materna conserve sus diámetros normales.

Puede ocurrir que una presentación inicial de frente, antes cie buscar encajamiento, adopte la actitud de deflexión parcial y que, en el transcurso del trabajo y gracias al impulso que le imprime el músculo uterino, por una parte, y la resistencia de los huesos pelvianos, por la otra, logre convertirse en presentación ce cara o de vértice evolucionado hacia un parto espontáneo.

Muy otra será la situación cuando la presentación de frente no se modifica, sino que, por el contrario, a expensas de contracciones musculares violentas se deforma y moldea la cabeza fetal para buscar encajamiento por el diámetro transversomaterno, que es el más amplio, porque entonces el parto se detiene y comienza la zona de peligro para la madre, si el segmento sobredistendido se rompe, y para el feto, si asciende el tono uterino con tetanización de la fibra por la mala oxigenación placentaria, determinante de la asfixia.

tes distocias dinámicas por inercia debida a la fatiga del músculo, 
Cuando el feto es prematuro y están por consiguiente reducídos los diámetros cefálicos, en relación con los de encajamiento, el. parto podrá efectuarse espontáneamente, aunque en forma laboriosa y prolongada. Si tal circunstancia no concurre, el parto será distócico y sólo realizable, con feto vivo, por vía abdominal o con basiotripsia con feto muerto.

En el servicio se siguió esta conducta: una vez diagnosticada por tacto vaginal la presentación de frente, con feto vivo y a término, se esperó el tiempo conveniente para apreciar un posible cambio en aquélla, por la consideración de que las contracciones uterinas normales sin inyección de hormona hipofisaria, poủrian legrar la deflexión total o la flexión de la cabeza, y de esta manera, ya transformada la presentación primitiva en una de cara o de vértice, aconsejar determinado procedimiento. Mas en vista cae la persistencia de la presentación de frente y al observarse ciue la bolsa de las aguas se conservaba con total o escasa dilatación cervical, se intentó la mutación por maniobra de Thorne previo rompimiento de las membranas y después de colocar a la paciente en Trendelemburg y someterla a anestesia. Ante el primer fracaso de la maniobra, se desistió para evitar la procidencia del cordón o de los miembros fetales y no causar desgarraduras en el segmento, y se apeló a los medios quirúrgicos para extraer el feto.

En sintesis, en la mayoria de los casos tuvo poco éxito el propósito de cambiar la presentación de frente por la de cara o vërtice, terminándose ordinariamente por asumir la conducta quirúrgica de la cesárea segmentaria.

En esta modalidad de presentación cefálica se desechó el procedimiento de la versión interna, por estimarse altamente peligroso para la madre y de gran mortalidad fetal.

\section{SITUACION TRANSVERSA}

Número total de situaciones transversas: 27.

Frecuencia: $0.76 \%$.

Situación transversa cuya conducta se resolvió por cesárea: 10 .

Situación transversa cuya conducta se resolvió por versión interna: 8.

Segundo feto de embarazo gemelar: 2 .

Fetos de $61 / 2$ a 7 meses: 4.

Fetos a término: 2. 
Situación transversa, cuya conducta se resolvió por embriotomía raquirea: 7 .

Situación transversa cuyo parto se realizó con feto muerto y macerado: 2 de manera espontánea.

La conducta que se adopte en la situación transversa depende de varios factores que debe tener en cuenta el obstetra para lograr éxito con las diferentes intervenciones que sea preciso ejecutar. Durante el embarazo, y principalmente al final de lá gestación, la situación transversa habrá de modificarse por maniobras externas, colocando posteriormente un cinturón eutócico que fije al feto en situación longitudinal, sin que importe cuál de los polos se adapta sobre el estrecho superior, pero favoreciendo siempre la presentación cefálica en cuanto sea posible.

Al iniciarse el trabajo la conducta por seguir es parecida o igual a la adoptada durante el último mes de embarazo, es decir, acondicionar el mayor eje fetal al diámetro longitudinal del útero. En estas condiciones es más difícil modificar la situación ciel feto, sobre todo en la primigestante, por haberse desencadenado las contracciones uterinas, que no permiten intentar la versión fuéra de su intervalo. Si el parto continúa evolucionando en el mismo sentido, aumentará el poder contráctil de la fibra muscular uterina, y por las condiciones propias de la paciente sobrevendrá la ruptura intempestiva de las membranas antes de que el cuello adquiera una dilatación apreciable. Con la salida brusca del líquido amniótico y por la mala adaptación del polo al estrecho superior, son frecuentes la procidencia del cordón y el prolapso de los miembros superiores fetales. Si se abandona el parto, se retraerán las paredes uterinas sobre el cuerpo fetal, que está doblado sobre sí mismo, y comenzará la distensión del segmento inferior hasta provocarse su ruptura, tanto más peligrosa en cuanto se trate de un feto a término. De este modo el parto se hace patológico, y se pone en grave peligro la vida de la madre, ya que la del hijo, por lo general, está perdida.

En los casos de fetos pequeños o macerados, se puede obtener un parto espontáneo por alguno de los mecanismos descritos (de Roeder, de Douglas y de Denman).

En el servicio se intentó con éxito, sobre todo durante el embarazo, la versión externa para lograr la corrección de la mala situación fetal. Ya en el trabajo, la versión externa dio resultado menos eficaz, y para practicarla, se tuvo en cuenta la integridad de la bolsa de las aguas. Después de la versión, se puso en decúbito lateral la enferma según la dirección de la cabeza fetal, y 
se esperó el encajamiento del polo cefálico. La versión por maniobras internas se realizó por indicación especial cuando se reunieron los requisitos para ejecutarla, es decir, dilatación completa del cuello, integridad de la bolsa de las aguas, matriz relajada y poco excitable, anestesia de la paciente, tamaño reducido del feto entre seis y siete meses, feto muerto y macerado, y segundo feto en los embarazos gemelares.

Sobre la versión interna, como conducta en las situaciones transversas, logré infundir en mis colaboradores inmediatos desconfianza y recelo, con fundamento en el fracaso de los operadores de circunstancia, y esto sirvió para disminuir los sucesos. Con todo, la versión interna contraindicada se realizó en dos casos por vicios de la organización hospitalaria, con los resultados que eran de esperarse, no propiamente porque el sistema sea en si mismo condenable, sino porque su aplicación es tan exigente y necesita condiciones tan precisas, que sólo por excepción resulta eficaz.

La cesárea segmentaria sigue siendo la conducta de predilección en la presentación distócida de hombro, cuando el feto está a término y vivo. No interesa, hoy en dia, el hecho secundario de la infección o de la probable contaminación del contenido uterino por la ruptura prematura o precoz de las membranas, pues el uso de los modernos antibióticos ha disminuído al máximo la morbilidad y mortalidad post-quirúrgica materna y en todo caso dicha conducta da un margen de seguridad mayor en relación con la vitalidad fetal.

Cuando el feto muere dentro del útero conservando su situación transversa, la operación indicada no es la versión interna, sino la embriotomía raquídea, que secciona el producto de la concepción simplificando su extracción, mediante la utilización del aparato de Torpin, de fácil manejo y de mayor seguridad que el uso de las tijeras de Dubois y el gancho de Browm.

\section{PLACENTA INFERIOR}

Observaciones de placenta inferior: 33 .

Variedad latero-marginal: 13.

Variedad central: 20.

Frecuencia de placenta inferior: $0.93 \%$.

Frecuencia de inserción latero-marginal: $0.36 \%$.

Frecuencia de inserción central: $0.57 \%$. 
En relación con la primera variedad es alto el porcentaje de mortalidad, pues sobre treinta y tres observaciones hubo dos defunciones que lamentar, y en relación con la segunda, es de tener $\epsilon$ cuenta el sinnúmero de partos prematuros que se ocasionan con la consiguiente repercusión en la morbilidad y mortalidad fetal.

Hay condiciones desfavorables en el medio hospitalario en gue me tocó actuar para un tratamiento eficiente, entre ellas la ignorancia de nuestras gentes humildes, que únicamente concurren a las salas de maternidad después de sufrir hemorragias de consideración por su cantidad, intensidad y frecuencia, que cuando no terminan con la vida de la paciente, la colocan en circunstancias de inferioridad orgánica que imposibilitan el tratamiento obstétrico inmediato y obligan a mejorar primero el precario estado de la salud con repetidas transfusiones de sangre total para recuperar el volumen del líquido hemático perdido. La demora para internarse la madre en un medio quirúrgico y la imperiosa necesidad de restaurar su estado general, repercuten indiscutiblemente en forma desfavorable sobre el producto de la concepción. De ahí que en algunas ocasiones me hubiera visto precisado a emplear maniobras de emergencia, no recomendables como conducta en todos los casos similares, sino aceptables como recurso obstétrico del momento.

Para relatar las conductas asumidas en el servicio, se necesita ciiferenciar la variedad de inserción viciosa placentaria, teniendo en cuenta que ésta es latero-marginal o central. Aclaro que, para el diagnóstico de placenta inferior, se tuvo en mira la posibilidad de palpar la inserción viciosa de la placenta, o en su defecto, el sintoma de hemorragia externa en la segunda mitad del embarazo o durante el parto, debido al desprendimiento por inserción viciosa. Las diferentes conductas fueron:

En la placenta latero-marginal el síntoma de hemorragia es el que da la pauta para el tratamiento. Por lo general las primeras hemorragias vaginales son tardías y se producen al final de la gestación, cuando la placenta se implanta lateralmente. En cambio, las hemorragias son más precoces en la inserción marginal. Después de internada la paciente en el medio quirúrgico, se debe valorar clínicamente y por exámenes de laboratorio el estado de anemia en que se halla la madre, factor que depende de la intensidad y frecuencia de las metrorragias y señala la conducta definitiva. Hay casos en que, al exponer a la madre a nue- 
vas sangrias de repetición, se la hace correr grave peligro, y entonces se debe provocar el parto para mayor seguridad materna y fetal, máxime si se atiende al hecho de que la hemorragia debió iniciarse después del séptimo mes de gestación, lo que garantiza ia viabilidad fetal en buen número de observaciones. Como tratamiento general es aconsejable prescribir las transfusiones de sangre en igual cantidad a la de la sangre perdida, y como conducta obstétrica valdría la pena de ensayar la ruptura amplia de la bolsa de las aguas para que disminuya la tracción que ejercen las membranas gruesas e inextensibles sobre la placenta en su colgajo inferior y asi no avance el desprendimiento que favorece la hemorragia. Además, durante el parto se añade a este mecanismo el deslizamiento del segmento sobre el polo inferior del huevo que, para Shroeder y Dumas, causaría la separación de la caduca básica inter-útero-placentaria, con la consecuente hemorragia externa. Con la ruptura de las membranas y la salida del líquido amniótico desaparecen estos factores de desprendimiento, y la presentación fetal busca encajarse comprimiendo la placenta que hace la hemostasis mientras se cumple el parto por las vías naturales. Si no obstante la ruptura amplia de la bolsa ce las aguas, continúa sangrando la herida útero-placentaria, no yesta sino la cesárea como única intervención obstétrica aceptable.

Cuando las hemorragias por inserción viciosa latero-marginal fueron de poca intensidad o frecuencia, sin que acuse la paciente fønómenos anémicos, se puede esperar la continuación del embarazo hasta la mayor viabilidad fetal, sedando el útero con medicación calmante, como opio, cloral, progesterona y betaglicerofosfato de sodio por vía endovenosa y mejorando la coagulación sanguínea, todo ello para evitar nuevas hemorragias por nuevos desprendimientos placentarios que obliguen a la intervención çuirúrgica.

Si apareciendo las hemorragias durante el parto, el cuello se dilata completamente para permitir el paso del feto y los diámetros de la pelvis guardan las debidas proporciones, deberá acelerarse la expulsión del feto aplicando el forceps, en la presentación cefálica, o hacerse extracción podálica en la presentación de pelvis.

En la diagnosis de una inserción placentaria, central, total o parcial, si la hemorragia pone en grave peligro la vida de la paciente, se debe intervenir siempre por cesárea segmentaria, después de mejorar el estado general con transfusiones copiosas de 
Sangre. La evacuación rápida del contenido uterino, con feto viable, es la única forma de asegurar la hemostasis de los vasos sangrantes.

Sobre treinta y tres observaciones de placenta inferior, se intervino por cesárea segmentaria en ventiún de ellas; se practicó versión interna en tres, y se resolvió el parto por las vías naturales en nueve pacientes.

\section{DESPRENDIMIENTO DE LA PLACENTA NORMALMENTE INSERTADA}

Observaciones de desprendimiento de placenta normalmente insertadas: 2 .

Frecuencia: $0.02 \%$.

Esta eventualidad puede acontecer entre el cuarto y el noveno mes de gestación, y casi siempre reconoce, como causa etiológica, la toxicosis gravídica, por lesiones vasculares que originan ci desprendimiento de la placenta.

Ordinariamente se presenta en las cercanías del término del embarazo, y en su sintomatología priman los fenómenos de shock $\mathrm{y}$ anemia por hemorragia mixta, interna primero en mayor grado $\mathrm{y}$ luégo externa al separarse las membranas. Pasa desapercibido el fenómeno cuando demuestra sintomatología benigna; pero cuando es de mediana intensidad, provoca hemorragias retroplacentarias que conducen al desprendimiento total, de características graves.

El tratamiento se debe orientar a combatir el estado de shock $y$ detener la hemorragia uterina tan pronto como fuere posible, antes que se modifique la constitución anatómica de las fibras musculares y se inicie la apoplejía útero-placentaria de Couvelaire. La única forma de contener la hemorragia uterina, que dio origen a la anomalía, es apresurar la evacuación del contenido uterino, y para ello ha de considerarse el período de la gestación en que ocurrió el desprendimiento.

Si el desprendimiento se realizó durante el embarazo o al iniciarse el parto, la laparotomía abdominal es la conducta de elección, como única manera de extraer con rapidez el feto y de poder apreciar la integridad funcional y anatómica de la musculatura uterina a fin de conservar el órgano, cuando se estime conveniente, o extirparlo en caso contrario.

El estado de shock impone vigilancia y medicación de uso corriente, y las manifestaciones anémicas hacen necesarias las transfusiones de sangre en suficiente cantidad. 
La histerotomía, en los casos graves de desprendimiento de la placenta, no estando lesionado el músculo uterino, es indispensable. La histerectomía, cuando existe apoplejía uterina, es la terapéutica profiláctica de las complicaciones de esfacelo y gangrena de la matriz alterada.

La ocurrencia, en el transcurso del parto, del desprendimiento de la placenta normalmente insertada, acompañada de dilatación avanzada del cuello, indica la rápida expulsión del feto por las vías naturales, mediante los diversos sistemas obstétricos.

Defiendo sin vacilaciones la conducta activa e inmediata cuando se diagnostica con certeza el desprendimiento placentario, porque no me convence cientificamente la espera indefinida funclada en el tratamiento sintomático del dolor y el shock mientras se efectúa el parto espontáneo, ya que si es verdad que éste se produce, ello ocurre a costa de la alteración intrínseca de las diversas capas del útero, debida al tiempo transcurrido en la expectativa, dando lugar unas veces a la estirpación quirúrgica del órgano y otras a la continuación de la hemorragia, que imposibilita toda intervención quirúrgica, reduciendo las funciones del cbstetra a la simple observación de la bondad de los medicamentos, con el posible reato de no haberse actuado quirúrgicamente en la forma impuesta por las condiciones especiales de la enferma.

El desprendimiento de la placenta normo-inserta es un accidente grave para la madre y mortal para el hijo, si se atiende a las estadísticas más numerosas de Bland y Rocoff, quienes sobre 2.319 observaciones dedujeron una mortalidad materna de $6.3 \%$ y una mortalidad fetal de $66.4 \%$.

\section{FORCEPS}

Número total de aplicaciones de forceps: 252.

Indicaciones: Falta de rotación y descenso: 68; Trabajo prolongado: 63; Sufrimiento fetal: 53; Profiláctico 23; Primigestante a.nosa: 2; Preeclampsia: 9; Eclampsia: 3; Inercia secundaria: 8; Cesárea anterior: 5; Procedencia del cordón: 2; Feto gigante: 2; Inminencia de ruptura del útero: 1; Cardiopatía: 1; Placenta previa: 1; Cara: 1; Edema de la vulva: 1; Frecuencia: $7.10 \%$; Muertes fetales: 25; Frecuencia de mortalidad fetal: $9.9 \%$; Frecuencia de mortalidad fetal corregida: 5.5\%.

Causas de la muerte del feto: Asfixia por procidencia del cordón: 2; Trabajo prolongado fuéra del servicio: 6; Eclampsia Materna: 1; Septicemia: 1; Macerado: 1; Neumonía: 1; Menin- 
gitis: 1; Forceps laboriosos: 4; Hemorragia craneana: 3; Forceps atraumático y muerte fetal: 5 .

Mortalidad materna: $0 \%$.

Las condiciones generales requeridas para una buena aplicación de forceps deben tenerse en todo instante presentes, so pena de incurrir en errores serios y de difícil reparación.

En las aplicaciones de forceps que se hicieron en el servicio, se empleó únicamente el modelo pequeño de Simpson por su fácil manejo operatorio, si bien la rotación se dificulta con este instrumento en las tomas clásicas, y es presiso acudir a diferentes manicbras obstétricas con el mismo aparato para lograrla.

Contraindica completamente la aplicación del forceps la desproporción cefalopélvica, que tampoco debe intentarse cuando se crea que el parto por las vias naturales, y mediante este instrumento, acarreará traumatismos graves para la madre y reservados o mortales para el feto.

Sus indicaciones terapéuticas son múltiples. Me limitaré a cescribir algunas de ocurrencia en el servicio:

Falta de rotación y descenso. - En términos generales, la inciicación del forceps provendrá de la incompleta, pervertida o deficiente rotación de la presentación, cuando se presume que la sctividad muscular espontánea es incapaz de producirla, y su funcíón es sólo complementaria de las fuerzas naturales.

Cuando quiera que la toma clásica no dio resultado en la rotación de las variedades posteriores o transversas, se tuvo éxito con la maniobra de Ramirez Merchán, que en gracia de la brevedad no se describe en el presente ensayo y que en la literatura médica colombiana y aun en la extranjera, ha alcanzado ya la categoría de un principio científico.

Para prevenir complicaciones del trabajo prolongado se utiliza el forceps, siempre que se llenen los demás requisitos indisrensables a su aplicación y la presentación no descienda en el canal genital.

En la práctica hospitalaria fue de frecuente osurrencia el ingreso de pacientes que iniciaron su trabajo con anterioridad, y la mala conducción del parto por comadronas ignorantes modificó y alteró la dinámica uterina, prolongando la duración de aquél.

Sufrimiento fetal.- En las civersas observaciones anotadas, al diagnosticarse el sufrimiento fetal en el transcurso del parto y al llenarse los requisitos para la aplicación del forceps, se procedió 
en forma inmediata a su empleo. Así mismo se utilizó este instrumento para extraer el feto, cuando la prolongación del trabajo influía sobre la vitalidad fetal, y se presumía el sufrimiento fetal inminente.

Forceps profilácticos.- Cuando la cabeza se halla en el periné o se hace visible en cada contracción uterina y, a pesar de los eqfuerzos expulsivos no se verifica el parto, o cuando el músculo se fatiga y deja de contraerse, o cuando la resistencia del suelo pelviano no se vence con una episiotomía amplia, se puede acelerar y facilitar la expulsión del feto por una aplicación de forceps, que se denomina profiláctica por no deberse a indicación urgente, sino a la voluntad del obstetra, que desea aminorar el sufrimiento cie la madre acelerando la expulsión, sin riesgo para la vida de la madre y el feto.

Preeclampsia y eclampsia.- En esta variedad de toxemia gravídica urge la extracción del feto, como única causa provocadora ce la entidad, y es de mayor necesidad cuando el tratamiento médico adecuado no da el resultado que se espera.

En la preeclampsia del parto, cuando aumenta la tensión, se acelera el pulso y se acentúa la cefalea en casco y la epigastralgia, se debe apresurar la extracción del feto por las vías naturales, si fuere posible, con el fin de evitar los ataques convulsivos de la eclampsia, que son de pronóstico más reservado para la madre y para el hijo. La extracción se efectuará con forceps, según las condiciones especiales de la paciente.

Inercia secundaria.- En la hipótesis de que el músculo se fatigue y la fibra muscular no responda a los ocitócicos, después de que las contracciones uterinas hubieren logrado la dilatación total o parcial del cuello, el médico asegurará la continuación del parto con el empleo de forceps.

Cesárea anterior.- Si el parto transcurre dentro de los límites normales y no se advierte desproporción cefalopélvica o distocia dinámica, se puede esperar la diltación del cuello que cermita el paso de la presentación cefálica en las pacientes sometidas previamente a intervención quirúrgica sobre el segmento inferior. Pero no es aconsejable, una vez encajada la cabeza, seguir sometiendo a prueba la resistencia de la pared uterina para aguardar un parto espontáneo, sino que es de lógica elemental favorecer la expulsión del feto mediante una aplicación ce forceps. 
Procidencia del cordón.- Al descender el cordón umbilical por delante de la presentación, se pone en grave peligro la vida del feto y su extradición rápida es la forma de actuar.

Al encontrarse el cuello completamente dilatado y si se facilita el descenso y la expulsión, el forceps puede introducirse para salvar la vida del hijo. Si estas condiciones no se cumplen, la cesárea segmentaria garantiza mayor rapidez en la evacuación del contenido uterino.

Feto grande.- El tamaño exagerado del feto motiva la desproporción cefalopélvica, contraindicando el uso del forceps, pero si la pelvis de la madre es amplia, puede lograrse el encajamiento de la presentación, aunque con dificultad en el descenso, artificialmente.

Cardiopatia.- No hay discordancia en los autores frente a la conducta en las lesiones cardíacas de la embarazada durante el parto. Todos aconsejan evitar los esfuerzos expulsivos mecliante el forceps.

Presentación de cara.- En la única paciente que fue objeto de aplicación de forceps para una presentación de cara profundamente encajada en MIIA, se alcanzó éxito en la rotación y extracción del feto. Las buenas contradicciones uterinas habian sido inútiles para el parto espontáneo.

Edema de la vulva.- En esta observación el parto transcurrió normalmente hasta llevar la presentación al estrecho inferior; vero las manipulaciones y táctos de una enfermera fuéra del servicio le provocaron a la paciente un edema traumático e infeccioso de la vulva que, a pesar de la episiotomía amplia que se practicó, impidió el paso de la cabeza fetal, haciendo necesaria la Litilización del forceps para lograr el parto.

Inminencia de ruptura del útero y placenta previa.- La aplicación de forceps en estas entidades busca únicamente acelerar el parto, para evitar la desgarradura del órgano, en el primer caso, y en el segundo, la continuación de la hemorragia.

\section{DATOS ESTADISTICOS}

En todas las aplicaciones de forceps se tuvieron en cuenta las indicaciones absolutas. De ahi que, en la gran mayoría de los casos, se obtuviera buen resultado. Se hicieron siete tomas por sobre el estrecho superior, con cabeza sin encajar, con sufrimiento fetal, sin desproporción cefalopélvica y cor dilatación completa 
del cuello. Todas las pacientes eran multíparas. En las aplicaciones restantes, se intervino con presentación cefálica encajada, a diferente altura de los planos paralelos de Hodge, con episiotomía paramediana en las primíparas. Para la estimación de la mortalidad fetal, justo es anotar que la aplicación de forceps, en la mayoría de las parturientas llegadas al servicio, tuvo por causa el avanzado sufrimiento fetal anterior a la hospitalización.

\section{VERSION INTERNA PODALICA}

Observaciones de versión interna podálica: 11.

Frecuencia: $0.31 \%$.

Mortalidad materna: $0 \%$.

Frecuencia de mortalidad fetal: $45.45 \%$.

Mortalidad fetal total: 7 .

La versión interna podálica es una maniobra obstétrica que tuvo gran auge para resolver dificultades en la práctica diaria, cuando se temía la intervención quirúrgica por las fallas operatorias y por las infecciones que solían producirse. Hoy, con los adelantos de la cirugía, no se justifica ese temor, y las indicaciones de la versión interna no sólo disminuyen considerablemente, sino que tienden a desaparecer del arte obstétrico en los centros médicos científicos.

La frecuencia con que se desgarra el útero durante la versión, infunde desconfianza limitando sus indicaciones. Nunca el operador queda satisfecho, pues duda una vez extraído el feto, de la integridad anatómica de la matriz, que continúa revisando después del alumbramiento por el alto porcentaje de rupturas uterinas que esta versión ocasiona, aun realizada con todos los requisitos indispensables a su buena ejecución.

Resumiendo, las tres únicas indicaciones de la versión interna son: a) Las situaciones transversas, cuando el parto acaece antes del término de la gestación, y por lo tanto el feto es pequeño, y cuando el cuello está completamente dilatado, y la bolsa sin romperse; b) Segundo feto de un embarazo gemelar, que se quiere extraer rápidamente por diversas causas feto-maternas $u$ ovulares, y c) Procúbito o procidencia reciente del cordón, con feto pequeño, cuando se reúnen los demás requisitos.

El hecho de lograr éxito en otras circunstancias, apenas indicará la buena suerte del obstetra que sometió la madre al peligro de la ruptura y el hijo a la muerte. En ninguna forma debe aceptarse la versión como conducta general, existiendo como 
existen otros sistemas que garantizan mayor supervivencia fetal con menor peligro de la paciente.

La versión interna está contraindicada en la tetanización o hipertonía uterina, por la resistencia que opone la pared a la mano del operador y por la imposibilidad de toda movilización. so pena de estallar el útero. Lo mismo acontece cuando el segmento adelgazado no puede distenderse más para que se deslicen las nalgas y pueda ascender la cabeza fetal. La hemorragia de la inserción viciosa de la placenta no permite la diltación completa del cuello, que es requisito previo indispensable para la versión; completarla artificialmente es favorecer la continuación de la hemorragia, desgarrando los tejidos cervicales. Mejor que la versión interna, es la cesárea intraperitoneal en estas condiciones. Otra contraindicación es el sufrimiento fetal, que obliga al obstetra a buscar la rápida evacuación del contenido uterino por versión interna, cuando el feto en presentación cefálica, es de término, pues ocurre en la práctica que el cuello está incompletamente dilatado, y mayor tiempo se invertiría en completar la dilatación y hacer la versión que en tratar a la enferma por vía quirúrgica. Además, las maniobras necesarias para la versión provocarían mayor sufrimiento, fenómeno que no se presenta en la nisterotomia segmentaria. Las presentaciones distócicas de frente 氵 cara en mento-posterior, favorecen la ruptura precoz de las membranas, con escasa dilatación del cuello y con ascenso del tono basal uterino, todo lo cual contraindica formalmente la versión interna. Finalmente, si existe una cicatriz previa en el segmento, todas las maniobras ejecutadas dentro del útero son más peligrosas aún por la disminución en la elasticidad y por el fácil desgarramiento de la cicatriz con los cambios de presentación fetal.

Si el feto muere dentro del útero, debe esperarse el parto espontáneo. A no ser que su expulsión se dificulte porque entonces. en presentación cefálica, se hace basiotripsia, y en situación transversa embriotomía raquidea. Es preferible desistir oportunamente de una versión interna y practicar otra operación obstétrica, que arrepentirse más tarde de haberla logrado a expensas de peligros ingentes para la madre y el hijo.

Las indicaciones de esta versión en el servicio fueron:

Situación transversa en el segundo feto de un embarazo gemelar: 3; Situación transversa con feto pequeño: 3; Sufrimiento fetal con embarazo de ocho meses y presentación cefálica: 1; Placenta inferior latero-marginal con feto muerto: 1; Placenta in- 
ferior latero-marginal con feto vivo: 1; Placenta inferior central con feto muerto: 1; Procidencia del cordón y feto a término: 1 ,

En relación con la madre, no hubo que lamentar ningún desgarro uterino, quizás por reunirse los requisitos para la versión. No así, si se tiene en cuenta el pronóstico fetal, por la abundante mortalidad, que fue la siguiente:

Versión con feto vivo: 4; Versión con feto muerto o que murió antes de los tres días: 5; Versión sobre feto muerto: 2.

Son frecuentes, después de la versión, los fenómenos asfícticos del hijo, que dejan como secuela focos neumónicos y de atelectasia. Las fracturas de los miembros fetales son de ordinaria ocurrencia por las tracciones que se ejercen en el momento de la expulşión, lo que aumenta el índice de morbilidad y mortalidad fetal.

\section{OPERACION CESAREA}

Número total de intervenciones: 149; Número de intervenciones en primíparas: 66; Número de intervenciones en multíparas: 83; Número de cesáreas iterativas: 27.

Causas de las cesáreas: Desproporción cefalopélvica: 36; Estrechez pélvica: 10; Placenta inferior: 21; Presentación de pelvis en primigestante: 5; Eclampsia: 12; Procidencia del cordón: 8; Procidencia de los miembros: 2; Situación transversa: 11; Presentación de cara: 8; Manchester Fothergil reciente: 1; Muerte habitual del feto: 1; Eritroblastosis fetal: 2; Fractura de los huesos de la pelvis: 1; Fibroma del segmento uterino: 1; Sufriniento fetal: 15; Distocia de partes blandas: 1; Trabajo prolongado con falta de encajamiento e inminencia de ruptura del útero, 10; Primigestante añosa: 4.

Número de fetos muertos al nacer, o que murieron antes de que la madre saliera de? hospital: 32.

Causas de muerte fetal ajena a la cesárea: 20; Riñón poliquístico: 1; Neumonía: 1; Meningitis: 1; Feto muerto por placenta inferior antes de operar la madre: 2; Aplicación de $\mathrm{Cu}$ rare a la madre que causó la muerte del feto: 1; Feto hidrocefálico: 1 .

Número de fetos muertos, cuya causa se le podría atribuir a la intervención quirúrgica o al motivo que engendró la distocia: 11 .

Frecuencia de la cesárea: $4.21 \%$.

Frecuencia de cesárea en primigestante: $1.86 \%$.

Frecuencia de cesárea en multigestante: $2.35 \%$.

Mortalidad materna: $1 \%$. 
Frecuencia de mortalidad materna: $0.66 \%$.

Mortalidad fetal: $11 \%$.

Frecuencia de mortalidad fetal: $7.38 \%$.

Las ventajas de la incisión sobre el segmento, y no sobre el cuerpo, se exponen a continuación:

1. Menor hemorragia durante el acto operatorio; 2a Mejor a.frontamiento de los bordes de la herida; $3^{\text {a }}$ Peritonización más efectiva y fácil de la sutura uterina, con menor peligro de adherencias; 4a Mejor reparación cicatricial de la herida, ya que se actúa sobre el tejido elástico y no sobre el contráctil, con menor riesgo de ruptura del útero en nuevos embarazos, y 5 a Menor morbilidad materna por infección del peritoneo, que cuando se presenta, se localiza en la pelvis sin generalizarse al abdomen.

La histerotomía segmentaria se hace en media luna de concavidad superior, para evitar lesiones sobre los vasos uterinos; y en el sitio más bajo, cerca del repliegue vesico-uterino, para dejar hacia arriba espacio suficiente que facilite la intervención en futuras cesáreas.

No es la incisión del segmento la manera de sortear cualquier situación obstétrica difícil, sino la forma perentoria de actuar ante las exigencias ovulares, maternas o fetales. Precipitarse a realizarla antes de que surja la indicación, sería un error tan grave como prolongar la espera para llegar a la cesárea en malas condiciones, malogrando su resultado.

En relación con los peligros de la operación cesárea sobre el segmento, se anotan: a) Los de infección, que se combaten por los diversos antibióticos conocidos, cuya aplicación profiláctica reduce la morbilidad materna; b) Los de hemostasis, que no ofrecen dificultad especial por las características del segmento; c) Los de embolía de aire o amniótica, que son frecuentes en las cesáreas corporales por el mayor calibre de los vasos sanguíneos seccionados, pero disminuyen cuando se interviene en el segmento; d) Los de ruptura del útero en embarazos y partos posteriores, que dependen de la técnica empleada para el afrontamiento de la herida uterina, lo mismo que de la curación por primera intención de la cicatriz, y e) Los de formación de adherencias intraperitoneales, que se reducen con la incisión del segmento, donde la peritonización a expensas del colgajo vesical y segmentario se hace fácilmente.

Las indicaciones de la cesárea segmentaria se ampliaron con las innovaciones técnicas propias del procedimiento, y con las de 
cirugía general, que mejoraron las estadísticas de morbilidad y mortalidad feto-materna.

\section{ABORTO}

Número de abortos tratados: 593; Número de abortos completos: 113; Número de abortos incompletos asépticos: 312 ; Número de abortos incompletos sépticos: 168; Infección post-aborto completo: 15; Post-aborto incompleto afebril: 525; Post-aborto incompleto febril: 83; Frecuencia de abortos: $12.28 \%$; Frecuencia ce aborto completo: 19.05\%; Frecuencia de aborto incompleto: $80.95 \%$; Mortalidad materna por aborto: $0.16 \%$; Mortalidad materna rectificada: $0 \%$.

No es posible establecer la frecuencia de los abortos espontáneos y de los provocados, por el permanente engaño de las pacientes, interesadas en ocultar toda responsabilidad en la interrupción de su embarazo, y por ser el interrogatorio médico la única forma de ordenar una estadística precisa al respecto.

\section{MORTALIDAD MATERNA}

Número de defunciones maternas: 16.

Causas: Eclampsia: 6;

Placenta inferior: 2;

Hemorragia del alumbramiento: 1 ;

Shock transfusional: 1;

Edema agudo del pulmón: 1;

Peritonitis post-cesárea en hidrocéfalo: 1;

Shock obstétrico: 1;

Insuficiencia cardíaca: 1 ;

Anemia aguda post-aborto: 1 ;

Apendicitis supurada. Peritonitis. Embarazo: 1;

Frecuencia de mortalidad materna: $0.32 \%$;

Frecuencia de mortalidad materna corregida: $0.26 \%$.

En la consideración de la mortalidad materna en el servicio, se debe tener en cuenta una serie de factores desfavorables en la clientela hospitalaria, no frecuentes en la clientela particular del obstetra.

A pesar de que funcionan en el Instituto de Protección Materna e Infantil varios consultorios para la vigilancia prenatal de todas las enfermas embarazadas, su falta de asistencia a ellos o su ignorancia sobre los peligros que acarrea la preñez, perjudican 
el control del embarazo. Las pacientes concurren a las salas de hospitalización en los estados más avanzados de intoxicación gravídica o de anemia aguda. Cuán distinta sería su situación con la atención periódica que se dispensa en los consultorios: la preeclampsia y la eclampsia, lo mismo que la placenta inferior, $c_{i}$ ue son las entidades obstétricas que arrojan anualmente mayor mortalidad materna en los diversos servicios, podrían ser preve.lidas en mejor forma.

Eclampsia. - El tratamiento quirúrgico, como conducta raciical, se empleó en cinco observaciones, lográndose feto vivo en cuatro de ellas, después de la cesárea. Las madres se encontraban profundamente intoxicadas por la gestosis, tres en estado de coma $\mathrm{y}$ dos con ataques convulsivos subintrantes. El embarazo era de termino e inicialmente se trataron médicamente, pero ante el fracaso se procedió a vaciar el útero quirúrgicamente para extraer el feto causante de la toxicosis. En una sola observación, en que el feto murió durante los ataques, se continuó con la medicación sedante de gardenal, inyecciones endovenosas de sulfato de magnesia al 20\%, hasta la aparición de signos de saturación medicamentosa, y soluciones dextrosadas al $50 \%$, para aumentar la diuresis y disminuir los edemas. Sin embargo, el resultado final fue también lamentable por la muerte de la paciente.

El tratamiento médico o quirúrgico en mujeres embarazadas o parturientas, cuando se establece la toxemia aguda con convulsiones o coma o con lesiones características en los diferentes órganos de la economía, es de pronóstico grave, si no mortal, en la gran mayoría de los casos. Mejores resultados se obtienen en la preeclampsia con el tratamiento médico, si se deja la intervención quirúrgica como último recurso ante el fracaso de la medicación establecida. Todos los cuidados durante el embarazo, y especialmente en los últimos meses de la gestación, se deben encaminar a prevenir las manifestaciones toxémicas antes de que se desencadene la eclampsia, cuya aparición es señal inequívoca del deficiente tratamiento médico o quirúrgico durante la preeclampsia.

En el servicio se trataron las diferentes manifestaciones de la preeclampsia a su debido tiempo, y en las observaciones de eclampsia se obtuvieron resultados funestos por el descuido de las pacientes en el curso del embarazo, ya que todas ellas llegaron al hospital intoxicadas, cuando con el tratamiento sólo era dable esperar efectos milagrosos.

Anemia aguda. - La perdida de sangre por hemorragia externa vaginal causó la defunción de otras enfermas que pade- 
cían de inserción viciosa de la placenta, de aborto incompleto - de alumbramiento retardado. Todas ellas llegaron al servicio después de sangrías copiosas sin tratamiento adecuado.

Peritonitis. - La infección generalizada del peritoneo se observó en dos enfermas: en la primera, después de una cesárea corporosegmentaria para extraer un feto hidrocéfalo, y en la segunda, después de una apendicitis perforada coexistente con un embarazo de cinco meses. El tratamiento médico establecido a base de diferentes antibióticos, no dio resultado, y la muerte de la madre sobrevino al poco tiempo de la intervención quirúrgica, lo que demuestra la virulencia de los gérmenes patógenos que provocaron la infección.

Siempre he tenido por norma que el corte quirúrgico sobre el cuerpo es más susceptible de infección que la incisión sobre el segmento, y si se considera que para la extracción de la cabeza cumentada de volumen por la hidrocefalia se requería en el caso aludido mayor amplitud de la herida uterina, la infección como consecuencia de la intervención era de temerse.

El embarazo favorece el recrudecimiento de un proceso apendicular crónico, por la mayor congestión que le imprime al apéndice la vecindad del aparato genital hipertrofiado e hipervascularizado. La sintomatología aguda que experimentaba la paciente se debía a la perforación del apéndice con formación de un plastrón adherido al útero. La evacuación artificial del abceso por laparotomía difundió la peritonitis, pero la intervención quirúrgica era la conducta para solucionar las condiciones patológicas en que se encontraba la enferma.

Insuficiencia cardíaca y edema agudo del pulmón.- Las lesiones cardiacas, por leves que sean, están sometidas a la descompensación durante el embarazo y principalmente en el parto y el puerperio. Todo depende de la capacidad funcional del miocardio. Las contracciones uterinas aumentan la tensión arterial y suscitan cambios tensionales que un corazón normal soporta, pero que un corazón lesionado no tolera sin que se altere su funcionamiento. Los esfuerzos expulsivos en el segundo período del parto, predisponen a la hiposistolia, a la asistolia, al colapso cardíaco y al edema pulmonar, por lo cual se deben evitar con aplicaciones de forceps que aligeren la expulsión del feto y prevengan estas complicaciones. 


\section{MORTALIDAD FETAL}

Número de nacimientos: 3.585 .

Número de niños nacidos vivos: 3.422 .

Número de mortinatos: 163.

Número de niños que murieron después del nacimiento: 154.

Frecuencia de mortalidad fetal al nacer: $4.54 \%$.

Frecuencia de mortalidad fetal después del nacimiento: $4.28 \%$.

Frecuencia de supervivencia fetal: $95.46 \%$. ,

Para valorar los indices de mortalidad fetal en los servicios hospitalarios, preciso es descontar el retardo de las madres en llegar al servicio por los motivos de que ya he hablado y, además. por el funesto perjuicio social que les hace preferir una asistencia doméstica insuficiente, cuando no dañina, a la atención científica en un hospital de caridad.

La muerte del feto, al final del embarazo, reconoce diferentes causas etiológicas: a) Las enfermedades infecciosas de la madre, cuando se acompañan de hipertermia intensa por disminución de la respiración tisular; b) Las infecciones crónicas, como la sífilis, la tuberculosis y la diabetes; c) Las gestosis gravídicas, como la preeclampsia y la eclampsia; d) La asfixia y anemia de la madre; e) Las malformaciones congénitas; f) La eritroblastosis fetal, y g) Las circulares y nudos falsos y verdaderos del cordón. que interrumpen la circulación feto-materna.

Tales fueron las causas de mortalidad fetal durante el embarazo. En el trabajo se añaden a las causas mencionadas la mala atención del parto fuéra del servicio, que prolongó su duración e hizo laboriosa la expulsión del feto, y la asfixia, que se provoca por las contracciones musculares sostenidas e intensas, sin períodos de descanso, que impiden la renovación de la sangre en los senos placentarios. Estas distocias dinámicas se causan por los ocitócicos mal empleados, cuando el parto se inicia en la casa $\jmath$ por el mecanismo de defensa del músculo para vencer la resistencia que la rigidez del suelo pélvico o la pelviciación ósea oponen a la salida del feto. Todo repercute en la prolongación del trabajo, y el peligro de muerte por asfixia guarda relación con el tiempo que dure la tetanización uterina. Además, la retracción violenta de las paredes del útero sobre la placenta menos elástica reduce la oxigenación fetal, favoreciendo los desprendimientos.

La asfixia paralítica que padece el hijo al nacer, es la consecuencia de las compresiones internas o externas del encéfalo, y 
fueron causa, en las experiencias del servicio, de mortalidad fetal durante el parto. Hay compresión externa cuando la peivis estrecha dificulta el paso de la cabeza fetal, cuando el periné fibroso y poco elástico demora la expulsión del hijo y cuando se hacen aplicaciones de forceps laboriosas o contraindicadas. Hay compresión interna local de los centros respiratorios y cardíacos cuando aumenta la tensión endocraneana por hemorragia interna o por fractura de los huesos de la bóveda cefálica fetal. La parálisis respiratoria puede sobrevenir cuando se administran a la madre, con exceso, los gases anestésicos, los barbitúricos, el demerol, la morfina, el curare $u$ otros medicamentos que rebajen la sensibilidad a los estímulos naturales del parto y de la vida. extrauterina.

Dentro de la mortalidad fetal en el servicio, se observó una mayor ocurrencia de asfixia por causas sofocantes que interrumpieron directamente el abastecimiento de oxígeno. Entre ellas merecen mención especial la procidencia del cordón y el trabajo prolongado.

Las causas de muerte fetal después del nacimiento, están ligadas, en su mayor parte, a la compresión anormal del cráneo fetal en su paso por el canal genital, que dio lugar a hemorragias cerebrales con fenómenos asfícticos. Los traumatismos graves con fisura, fractura o desgarro de las suturas inter-óseas, no son privilegio exclusivo de los partos intervenidos con forceps; también se presentan en los nacimientos espontáneos, cuando las contracciones musculares adquieren suficiente intensidad y violencia para vencer la resistencia del cinturón óseo viciado o del canal vaginovulvar estrecho. En muchas ocasiones el sufrimiento fetal por trabajo prolongado indica la hemorragia cerebral y la aplicación cel forceps para acelerar el parto aparece como única causa provocadora de la lesión.

Los niños que nacen antes del término de la gestación, ofrecen marcada ocasión a las hemorragias meníngeas, por mayor ¿cabalgamiento de los huesos del cráneo y por mayor fragilidad de los capilares sanguineos.

La prematurez fue otra de las causas de mortalidad infantil después del nacimiento, siendo mayor la posibilidad de supervivencia cuanto más se aproxime la edad fetal al término del embarazo. La atelectasia, con estasis capilar y hemorragia pulmonar extensa, dio lugar a cianosis, disnea duradera y debilidad cardiaca. Cuando esta sintomatología se resistía al tratamiento, causaba la muerte de los niños prematuros y débiles congénitos. 
Las afecciones pulmonares por aspiración del líquido amniótico, meconio o sangre, fueron las complicaciones tardías de los partos demorados y laboriosos. En varias ocasines se obtienen resultados favorables con los antibióticos, pero las estadísticas demostraron frecuencia elevada de mortalidad fetal por neumorías o bronconeumonías del recién nacido.

\section{DATOS ESTADISTICOS}

Número de historias consultadas . . . . . . . . . . . . . . 4.951

Número de enfermas que salieron en el mismo estado $\begin{array}{lllllllllll}\text { obstétrico } & \ldots & \ldots & \ldots & \ldots & \ldots & \ldots & \ldots & \ldots & \ldots & \ldots\end{array}$

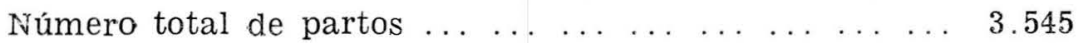

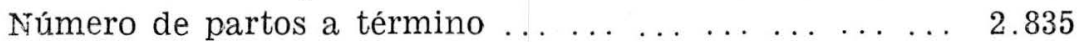

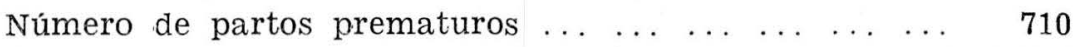

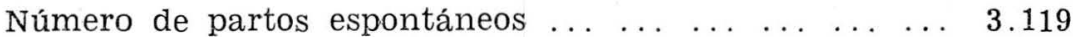

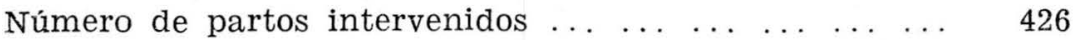

Parto en presentación de pelvis completa ... ....... 100

Parto en presentación de pelvis incompleta ... ... ... 50

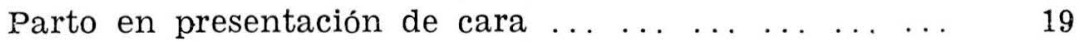

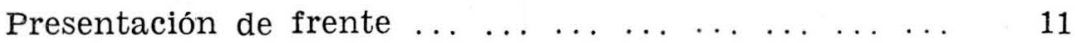

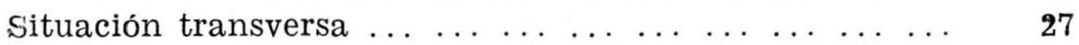

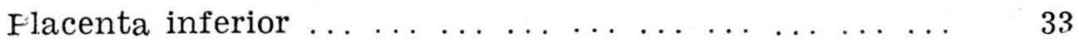

Variedad latero-marginal ........ $\quad . \quad 13$

Variedad central ... ... .......... 20

Desprendimiento de la placenta normalmente insertada 2

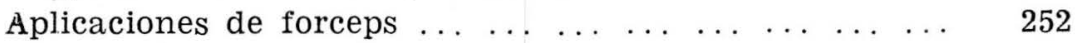

$\begin{array}{llllllllllll}\text { Fimbriotomías } & \ldots & \ldots & \ldots & \ldots & \ldots & \ldots & \ldots & \ldots & \ldots & \ldots & 10\end{array}$

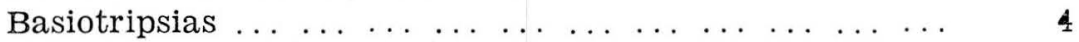

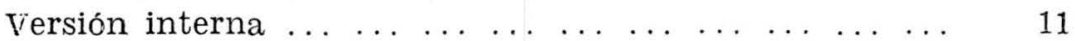

$\begin{array}{llllllllllllll}\text { Abortos } & \ldots & \ldots & \ldots & \ldots & \ldots & \ldots & \ldots & \ldots & \ldots & \ldots & \ldots & \ldots & 593\end{array}$

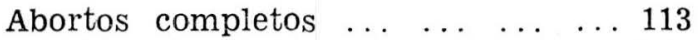

Abortos incompletos asépticos ... 312

Abortos incompletos sépticos ... . . 178

Infección post-aborto completo ... 15

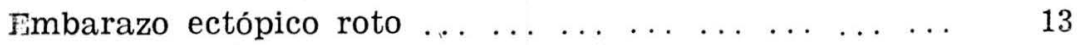

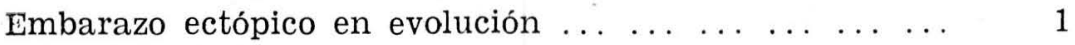

$\begin{array}{lllllllllll}\text { Mola hidatidiforme } & \ldots & \ldots & \ldots & \ldots & \ldots & \ldots & \ldots & \ldots & \ldots & 9\end{array}$

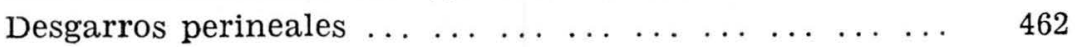




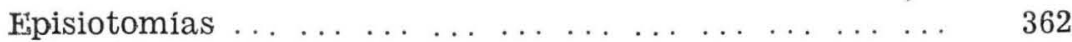

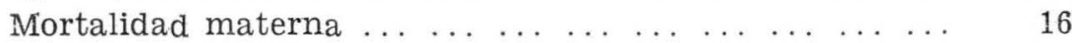

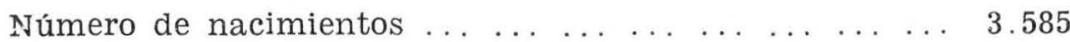

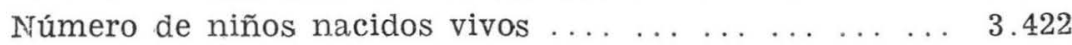

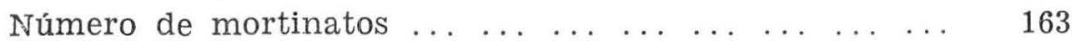

Número de niños que murieron después del nacimiento 154

\section{FRECUENCIAS}

Frecuencia de la presentación pelviana completa ... $3.21 \%$

Frecuencia de la presentación pelviana incompleta ... $1.60 \%$

Frecuencia de presentación de cara ... . . . . . . . . $0.53 \%$

Frecuencia de presentación de frente ........... . $0.31 \%$

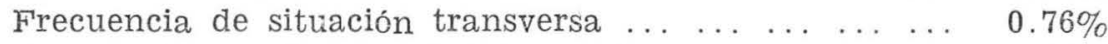

Frecuencia de placenta latero-marginal ... . . . . . . $0.36 \%$

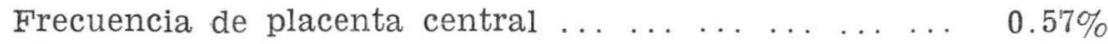

Frecuencia de placenta inferior ... . . . . . . . . . $0.93 \%$

Frecuencia del desprendimiento de la placenta normal-

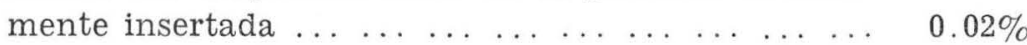

Frecuencia de aplicaciones de forceps . . . . . . . . . . $7.10 \%$

Frecuencia de versión interna podálica ... ... . . . . $0.31 \%$

Frecuencia de operación cesárea ... . . . . . . . . . . . $4.21 \%$

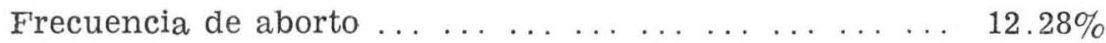

Frecuencia mortalidad materna total . . . . . . . . . . $0.32 \%$

Frecuencia de mortalidad materna corregida ... . . . . $0.26 \%$

Frecuencia de mortalidad fetal al nacer ... . . . . . .. $4.54 \%$

Frecuencia de mortalidad fetal después del nacimiento $4.28 \%$

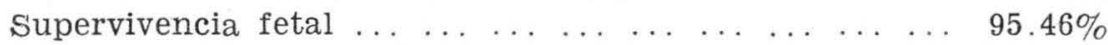




\section{BIBLIOGRAFIA}

Estadistica del Instituto de Protección Materna e Infantil de Bogotá.

\section{AUTORES NACIONALES}

Capasso Camilo.- "Consideraciones sobre 3.000 partos". Rev. Col. de Obt. y Gin. Vol. III. Nos. 3 y 4.

Corral Maldonado Jaime.- "Las grandes intervenciones obstétricas" (estudio clínico y estadístico en dos años). Rev. Col. de Obst. y Gin. Vol. II. Nos. 2 y 3.

Ramirez Merchán Rafael F.- "Conductas obstétricas controvertibles". Rev. de la Fac. de Med. U. N. Vol. XVI. Nos. 7 y 8.

Madriñán H. Danilo.- Tesis de grado. 1946. "Algunas urgencias de Clínica Obstétrica".

Ramírez Merchán Rafael F. y Lamus Guerrero Sergio.- "Aspectos del ejercicio obstétrico en la clientela civil”. Rev. Col. de Obst. y Gin. Vol. II. № 4. Echeverry Parra Gonzalo.- Tesis de grado. 1944. "Consideraciones clínicas y estadísticas sobre operación cesárea".

Parra Agustin.- Tesis de grado. 1946. "La presentación de frente".

Medellin Julio C.- Tesis de grado. 1946. "La presentación de hombro (estadística y clínica)".

Afanador Jaime.- Tesis de grado. 1945. "La placenta inferior en nuestro medio hospitalario".

\section{AUTORES EXTRANJEROS}

De Lee Greenhill.- Principios y prácticas de Obstetricia. 1945. Ed. Utea.

Nubiola Zárate.- Tratado de Obstetricia. Ed. Labor. 1951.

W. Stroeckel.- Tratado de Obstetricia. Edit. Modesto Uson. 1945.

Moragues Bernat.- Clínica Obstétrica. Edit. El Ateneo. 1946.

H. Finkelstein.- Tratado de las enfermedades del lactante. Edit. Labor. 1950.

Moscaro-Porcar José María.- Breviario de la Urgencia Obstétrica. Salvat. Editores. 1945.

Maygrier y Schwaab.— Manual de Obstetricia. Espasa Calpe. 1943. 